\title{
A Deformable Model for Bringing Particles in Focus
}

\section{Dahl, Anders Lindbjerg; Jørgensen, Thomas Martini; Larsen, Rasmus}

\section{Published in:}

Computer Vision, Imaging and Computer Graphics. Theory and Applications

Link to article, DOI:

10.1007/978-3-642-25382-9_6

Publication date:

2011

Link back to DTU Orbit

Citation (APA):

Dahl, A. L., Jørgensen, T. M., \& Larsen, R. (2011). A Deformable Model for Bringing Particles in Focus. In Computer Vision, Imaging and Computer Graphics. Theory and Applications: Computer Vision, Imaging and Computer Graphics. Theory and Applications Computer Vision, Imaging and Computer Graphics. Theory and Applications International Joint Conference, VISIGRAPP 2010, Angers, France, May 17-21, 2010 (pp. 81-95). Springer. Communications in Computer and Information Science No. 229 https://doi.org/10.1007/978-3-64225382-9_6

\section{General rights}

Copyright and moral rights for the publications made accessible in the public portal are retained by the authors and/or other copyright owners and it is a condition of accessing publications that users recognise and abide by the legal requirements associated with these rights.

- Users may download and print one copy of any publication from the public portal for the purpose of private study or research.

- You may not further distribute the material or use it for any profit-making activity or commercial gain

- You may freely distribute the URL identifying the publication in the public portal 


\title{
A Deformable Model for Bringing Particles in Focus
}

\author{
Anders Lindbjerg Dahl ${ }^{1}$, Thomas Martini Jørgensen ${ }^{2}$ and Rasmus Larsen ${ }^{1}$ \\ 1 DTU Informatics, Technical University of Denmark, Lyngby, Denmark \\ abd@imm.dtu.dk, \\ WWW home page: http://www.imm.dtu.dk/ abd \\ 2 DTU Fotonik, Technical University of Denmark, Ris $\varnothing$, Denmark
}

\begin{abstract}
We provide a deformable model for particle analysis. We investigate particle images from a backlit microscope system where particles suffer from out-of-focus blur. The blur is a result of particles being in front or behind the focus plane, and the out-of-focus gives a bias towards overestimating the particle size. This can be handled by only including the particles in focus, but most of the depicted particles will be left out of the analysis, which weakens the statistical estimate of the monitored process. We propose a new method for particle analysis. The model incorporates particle shape, size and intensity, which enables an estimation of the out-of-focus blur of the particle. Using the particle model parameters in a regression model we are able to infer $3 \mathrm{D}$ information about individual particles. Based on the defocus information we are able to infer the true size and shape of the particles. We demonstrate the capabilities of our model on both real and simulated data, and our approach shows promising results for a reliable particle analysis. The potential is more process information obtained over shorter sampling time.
\end{abstract}

Keywords: particle analysis, deconvolution, depth estimation, microscopic imaging

\section{Introduction}

Knowledge about individual particles can be essential in industrial process optimization. We address the problem of analyzing images of individual particles. Application examples include suspended particles in for example a fermentation process, oil droplets in water, coal particles in a power plant, and spray particles in air. Two image examples are given in Fig. 1. A vision-based system can provide knowledge about particle distribution, size and shape, and these parameters are often essential for system design or process control. Here the choice of the analysis method and the quality of the images are essential elements, so both analysis method and image acquisition should be chosen carefully. 


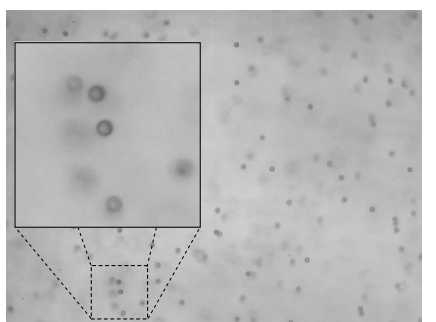

(a)

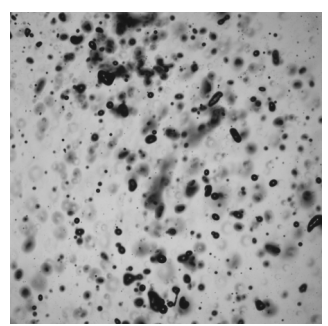

(b)

Fig. 1. Examples of particle images. (a) spherical transparent particles all $25 \mu \mathrm{m}$ in diameter, and (b) a typical image to be analyzed depicting spray particles.

\section{$1.1 \quad$ Motivation}

The motivation of our work is an industrial endoscopic inspection system equipped with a probe that can be placed inside the process ${ }^{3}$. Images are acquired from the tip of the probe, which also contains a light source placed in front of the camera. The resulting camera setup depicts particles as shadows, see Fig. 1. The visual appearance of the particles depends on the optical properties of the camera setup, the distance of the particles to the focus plane, and the physical reflectance properties of the particles. The depth of field of the camera optics is narrow and the particles get blurred as they move away from the focus plane, which introduces uncertainty of the particle characterization, c.f. Fig. 2. A tendency for overestimating particle size is reported in [11]. Employing a strategy where only in-focus particles are analyzed can be a good solution $[5,10]$, but in situations with few particles or short inspection time this approach will give an uncertain estimate due to low sample size. Therefore it can be necessary to perform the analysis of the blurred particles as well.

\subsection{Related work}

Our particle analysis approach combines the two elements of deblurring and shape characterization. The three dimensional nature of our problem does not allow the image acquisition system to have all particles in focus. Therefore we need to handle the out-of-focus blur, but this also provide information about the depth of the particles, which we use for estimating the spatial information about the particles. We will now address related work on deblurring followed by a discussion on particle shape modeling.

Deblurring In a linear system the image formation can be described as the linear convolution of the object distribution and the point spread function (PSF).

\footnotetext{
$\overline{{ }^{3} \text { PROVAEN }}$ - Process Visualisation and Analysis ENdoscope System (EU, $6{ }^{\text {th }}$ Framework)
} 


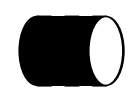

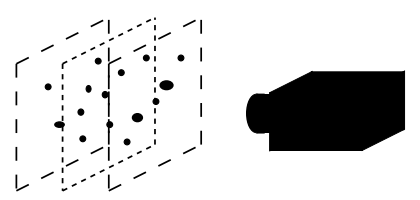

(a)

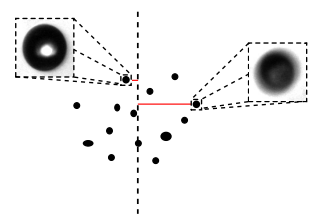

(b)

Fig. 2. Illustration of the particles relative to the focus plane. (a) particles in the 3D volume. (b) illustration of appearance change as a function of the distance to the focus plane.

Hence, to reduce the blur from out-of-focus light, ideally the mathematical process of deconvolution can be applied. However, noise can easily be enhanced if one just implements a direct inverse operation, so the inverse has to be regularized. Different regularizers can be employed, for example iteratively deconvolving the image [14], [16], or using a Wiener filter [20]. Alternatively, a maximum entropy solution can be chosen, which aims at being mostly consistent with data [15], [19]. These methods assume a known PSF. When this is not the case, blind deconvolution can in some cases be applied recovering both the PSF and the deconvolved image. Typically this is solved by an optimization criterion based on known physical properties of the depicted object [9].

These methods are based on the assumption of a known - possibly spacedependent - PSF for the image. For many optical systems it is difficult to calculate a theoretical PSF with sufficiently accuracy to be used for deconvolution. Also it can be quite difficult to measure it experimentally with sufficient resolution and accuracy. In our case the particles of concern are illuminated from the back and in this respect it resembles the case of bright light microscopy. Such an imaging system is not exactly a linear device but in practice it is almost so. However, in the bright field setting the "simple" PSF is compounded by absorptive, refractive and dispersal effects, making it rather difficult to measure and calculate it.

One method for local image deblurring, which is needed for our problem, include iteratively estimating the blur kernel and updating the image accordingly in a Bayesian framework [17]. Another approach is to segment the image and estimate an individual blur kernel for the segments $[2,12]$. Blur also contains information about the depicted objects. This has been used by $[4,18]$, where they obtain motion information by modeling blur. With a successful deblurring, e.g. based on one of these methods, we will still have to identify the individual particles. Instead, we suggest here to build a particle model.

Particle modeling Most particles have a fairly simple structure, typically being convex and close to circular or elliptical. This observation can be used for designing a particle model. In [6] a particle model is build for nanoparticles based on images obtained from an electron microscope. An elliptical model is aligned with the particles by maximizing the contrast between the average intensity of the 
particle and a surrounding narrow band. Particles in these images are naturally in focus.

Ghaemi et al. [7] analyze spray particles using a simple elliptical model. However, only in-focus particles are analyzed, and out-of-focus particles are pointed out as a cause of error. In addition, they mention the discretization on the CCD chip to be problematic, and argue that particles should be at least 40-60 pixels across to enable a good shape characterization. Blaisot and Yon [1] analyze diesel spray particles based on an image model derived from the point spread function for individual particles. They detect individual particles and detect the average particle diameter by applying a threshold based on the maximum and minimum intensity in a window around the particle. Furthermore they compensate for the out-of-focus blur by two additional thresholds, which they use for estimating the points spread. Furthermore they introduce morphological shape characterizations, that describes how much the shape deviates from a sphere.

We employ a deformable particle model for characterizing particles and account for out-of-focus blur. The parameters of our model encodes the shape of the particle in a natural manner. Based on the assumption that images are smooth we a able to obtain reliable shape and size information from particles smaller than 40-60 pixels in diameter. The main focus of this paper is our particle model, which we use for characterizing particle shape, size and blur. In Section 3 we describe our particle model and how it can be used for particle characterization. We experimentally validate the particle model in Section 4. Lastly, in Section 5 we discuss the obtained results, and we conclude the work in Section 6 .

\section{Contribution}

This paper is an extended version of our previous paper [3], where we demonstrated that particles size, shape and their distance to the focus plane reliably can be inferred using our deformable model. The contributions of this paper are

1. A modified and more robust particle model.

2. A higher level of detail in the model description.

3. Demonstration of the model performance on spray data.

These extensions makes the model more applicable for particle characterization and easier to implement and use in practical applications.

\section{Method}

The goal of the proposed method is to obtain information about the true size and shape of an out-of-focus particle. Our idea is to learn particle appearance from observations of particles with known position relative to the focus plane. By comparing the appearance of an unknown particle to the training set, we can predict how the particle would appear, if it was in focus. As a result we obtain information about the true particle size and shape. 


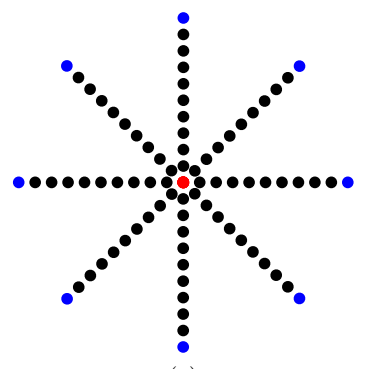

(a)

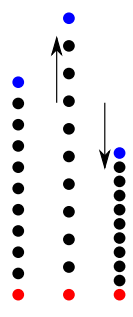

(b)

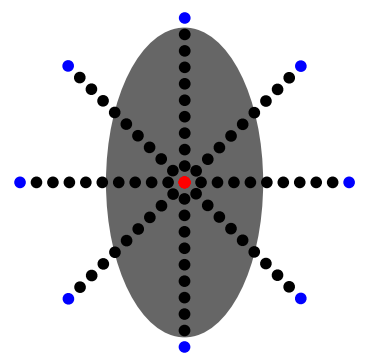

(c)

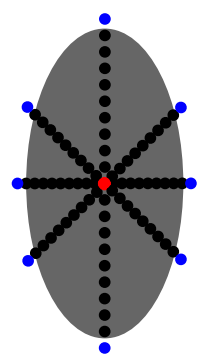

(d)

Fig. 3. Intensity sampling with the particle model. Radial sampling pattern of our model with 10 sampling steps from the center point, marked with red, to the end of the radial line marked with blue. There are 8 radial sampling lines in this example (a). Each radial sampling line can be deformed by stretching or compressing the line while keeping equal distance between the sampling points (b). This stretch of the individual sampling lines is what deforms our model. In (c) the model is placed over a particle and in (d) the model is deformed by changing the sampling lines to fit the particle.

To facilitate this, the particles must be characterized in a way that describes the appearance as a function of blur well. Furthermore, particles should be easy to compare. We will now give a short description of how particles are depicted, and then explain the details of our particle model and descriptor. Finally we describe the statistical model for depth estimation.

Experimental setup The particle analysis is based on backlight where the particles appear as shadows. Real image examples are shown in Fig. 1. In Fig. 2 the experimental setup is illustrated. Notice that all particles in Fig. 1(a) are the same size of $25 \mu \mathrm{m}$, but the blur makes them appear very different. Out-of-focus blur occurs both in front and behind the focus plane, but it is hard to tell if an observed particle is in front or behind, because the blur looks the same. As a consequence we have chosen to model the particles as a function of absolute distance to the focus plan, which is shown in Fig. 2. In Section 4 we experimentally show that these are reasonable assumptions.

Particle analysis model The objective is to design a model that encodes information about the particle's size, shape and blur. Our model is based on the observation that particles show close to radial symmetry up to scaling. If we sample along line segments from the center of the particle, we expect to see the same intensity pattern or a scaled version of this pattern. This is the idea that we base our particle model on, which is illustrated in Fig. 3.

Our particle prediction is based on the following

$$
\mathbf{Y}=\left[s_{t}, r_{t}, d_{t}\right]^{T}=f\left(c_{o}, r_{o}, I_{o}\right),
$$

where $\left(c_{o}, r_{o}, I_{o}\right)$ are the observed spatial position, shape and image appearance respectively, $f$ is the function mapping observations to the vector $\mathbf{Y}$ containing 

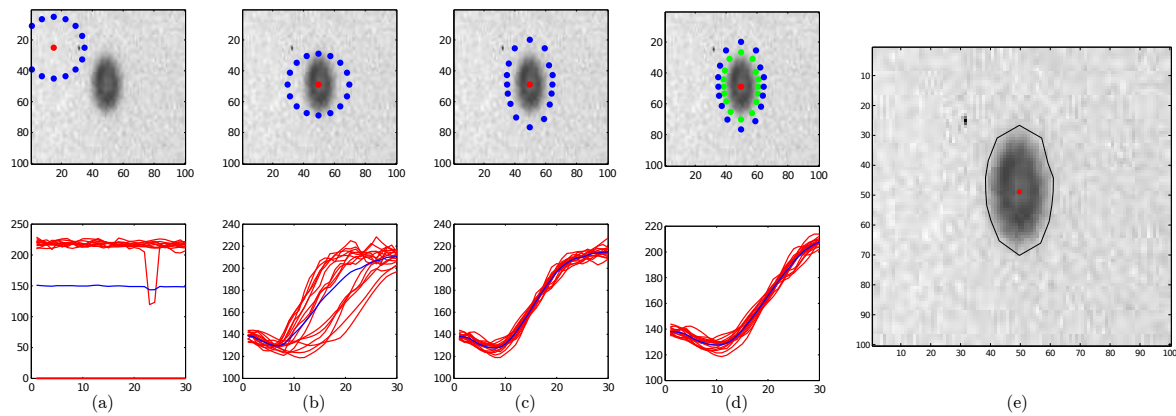

Fig. 4. Particle alignment and deformation. The image shows the particle, the red dot is the center, and the blue dots are the radial endpoints. The red curves show the intensity pattern along the individual radial lines and the blue is the average. The blob is initialized in (a), translated in (b), deformed in (c), the size is found in marked with green points (d) resulting in the segment in (e). Note that despite a very poor initial alignment the model finds the object very precisely. Also note how uniform the intensity pattern becomes by deformation.

the model prediction $\left(s_{t}, r_{t}, d_{t}\right)$ of size, shape and distance to the focus plane, respectively. We will now give the details of the particle model and then explain how the parameters of this model are used for predicting the particle characteristics.

We sample $n$ radial lines form the center coordinate $c_{o}$ placed with equal angle around the center point. A particle descriptor is obtained by sampling the image intensity along these radial lines at $m$ equidistant positions relative to the lengths of the radial lines. This intensity descriptor is denoted $I_{o}$. The length of the radial lines are stored in the $r_{o}$ vector, which characterizes the particle shape.

Alignment with image data Adapting the model to the image observations is done in the following four steps:

1. Detection of particles based on scale space blob detection [13].

2. Rigid model translation - the radial lines are not changed.

3. Coarse radial alignment using radial threshold.

4. Radial alignment using minimum intensity difference.

The particle model has to be initialized by a rough estimate of the particle size and position, and we have chosen to use scale space blob detection, see [13]. For computational reasons other blob-detectors could also be chosen. The blob detection supplies a subset of interest points in the image, and the rest of the image processing will take place around these points. As a preprocessing step for removal of noise we convolve the image with a Gaussian kernel with standard deviation $\sigma$.

Blob detection gives a particle center position, based on the response of an isotropic Laplacian operator. Many natural particles are anisotropic, so the cen- 


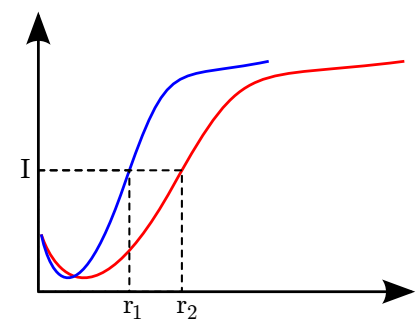

Fig. 5. Estimating the radial scale using threshold. The two curves illustrates two radially sampled lines, where one is a scaled version of the other. The horizontal axis is radial distance and the vertical is image intensity. The areas of the rectangles spanned by $\left(I, r_{1}\right)$ and $\left(I, r_{2}\right)$ are proportional to the scale between the two curves. We employ a threshold to find this proportion, and we weigh the thresholded values in the radial direction to account for image noise.

ter position can be inaccurate. To account for this inaccuracy we change the particles position by employing an optimization criterion based on radial symmetry and intensity variance. The reasoning for the first criterion is that particles are typically radially symmetric. Based on that we initiate our particle model with radial lines of equal length. We expect the radial lines to have highest similarity when they are sampled from the particle center, also for anisotropic particles. The variation criterion is based on the fact that the intensity descriptor has high variation when sampled on a particle and low otherwise. This turns out to be very important for the robustness of the alignment. The minimization problem becomes

$$
\underset{c}{\operatorname{argmin}}\left(\eta\left(\sum_{i=1}^{n}\left\|I_{i}-\bar{I}\right\|\right)-\xi \sigma_{\bar{I}}\right)
$$

where $\bar{I}$ is the mean intensity descriptor, and the sum of normed descriptor differences is weighed by $\eta . \sigma_{\bar{I}}$ is the standard deviation of the mean descriptor, which is weighed by $\xi$. This alignment is optimized using simple gradient decent, by moving in the steepest decent direction until an optimum is reached. The procedure is repeated with finer step size, until a desired precision is obtained. After an optimal particle position has been found, the particle shape is optimized to the image data by changing the length of the radial sampling lines. We employ two steps, to obtain a precise alignment. First we observe that the radial intensity samples are scaled in the radial direction and we employ a simple procedure to identify the scale parameter, which is illustrated in Fig. 5. This is baed on thresholding the radially sampled intensity values. We want to find the relative scale between two intensity curves $r_{1}=\alpha r_{2}$. First we estimate

$$
\alpha_{i}^{*}=\sum_{j=1}^{m} G(j) \hat{I}_{j}
$$


where $j$ is the number of sampling steps, $G(j)$ is the normalize Gaussian where $G(j)=\gamma \exp \frac{-j^{2}}{2 s^{2}}$ and $\gamma$ is chosen such that $\sum_{j=1}^{m} G(j)=1 . s$ is chosen relative the number of radial sampling points $(s=0.5 m) . \hat{I}_{j}$ is a thresholded intensity sample where

$$
\hat{I}_{j} \in\{0,1\}, \quad \hat{I}_{j}=0 \forall\left\{j \mid I_{j}>t\right\} \quad \wedge \quad \hat{I}_{j}=1 \forall\left\{j \mid I_{j} \leq t\right\},
$$

and $t$ is a threshold value chosen as the mean intensity $\bar{I}$ over all sample lines. $I_{j}$ is the sampled radial intensity. The scale $\alpha_{i}$ is found by

$$
\alpha_{i}=n \frac{\alpha_{i}^{*}}{\sum_{i=1}^{n} \alpha_{i}^{*}} .
$$

The threshold based shape alignment is repeated five times, to obtain a good alignment. After this initial alignment the radial sample lines are adjusted

$$
\underset{r}{\operatorname{argmin}}\left(\sum_{i=1}^{n}\left\|I_{i}-\bar{I}\right\|+\kappa \sum_{i=1}^{n} \sum_{j \in N}\left\|r_{i}-r_{j}\right\|\right),
$$

hereby minimizing the difference between the average descriptor and the individual radial descriptors, but taking length of neighboring radial lines $r_{i}$ and $r_{j}$ into account, where $N$ is the two neighboring radial lines. A large value of the weight parameter $\kappa$ will push the shape towards a sphere. This optimization is done similarly to the positioning, also using gradient decent and refining the step size when a minimum is reached. The length of the final radial lines are normalized to sum to the same as original radial lines lengths.

The particle model results in an observed characterization as follows

$$
\mathbf{x}=\left\{c_{o}, r_{o}, I_{o}\right\}
$$

containing the center position denoted $c_{o}$ which is a $2 \mathrm{D}$ vector, the length of the radial line segments denoted $r_{o}$ which is a $n$-dimensional vector, and the intensity pattern $I_{o}$ which is $m$-dimensional. It is estimated as the mean $I_{o}=$ $\frac{1}{n} \sum_{i=1}^{n} I_{i}^{\prime}$, where $I_{i}^{\prime}$ is the radial pattern of line segment $i$. It should be noted that the difference between the line patterns have been minimized, so we model the remaining difference as noise, and as a result the averaging will smooth this noise and make the estimate robust.

Modeling the particle will create an independent characterization of the size, shape and blur, which is illustrated in Fig. 3. Particle shape is encoded in the length of the radial line segments, and the particle size can be obtained from a combination of the radial intensity pattern and the length of the line segments. The intensity pattern $I_{o}$ has a shape that bends off to become indistinguishable from the background, see Fig. 4, and the particle boundary is estimated at this point. We found a function of the total variation to be good way of estimating this. We estimate the total variation as the sum of absolute differences of $I_{o}$ and we obtain the distance as 


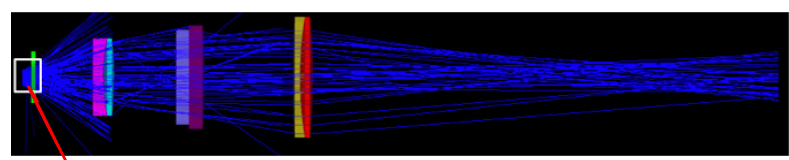

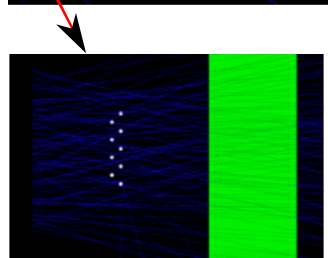

(b) (a)
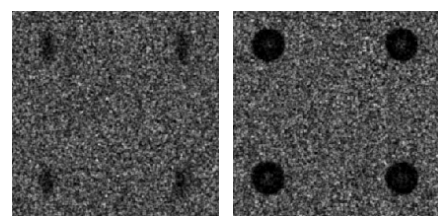

(c)

Fig. 6. Optical simulation in Zemax. (a) back illumination with a diffuse light source of $2 \mathrm{~mm}^{2}$ with wavelengths of 480-650 $\mathrm{nm}$ with transparent particles. (b) zoom on the particles and (c) examples of $50 \mu \mathrm{m}$ out-of-focus ellipsoid particels $(50 \mu \mathrm{m} \times 16.7 \mu \mathrm{m})$ and spherical in focus particles $(50 \mu \mathrm{m})$.

$$
\begin{aligned}
& r_{o}=\underset{j}{\operatorname{argmax}}\left(\frac{\sum_{i=1}^{m-j}\left|I_{o_{i}}-I_{o_{i+1}}\right|-c}{j \sum_{i=1}^{m-1}\left|I_{o_{i}}-I_{o_{i+1}}\right|}\right), \\
& j \in\{1, \ldots, m-1\},
\end{aligned}
$$

which is the normed total variation. The constant $c$ influences the estimated size of the particle.

Statistical analysis The blur is encoded in the radial pattern descriptor $\left(I_{o}\right)$, which we use as input for estimating the distance to the focus plane. We use a linear ridge regression to obtain the depth. The model is $d_{f}=I_{o} \beta^{r}$, where $\beta^{r}$ is the coefficients of the regression model. We obtain the model parameters from a training set with known distance to the focus plane by solving $\beta^{r}=$ $\left(I_{o}^{T} I_{o}+\lambda \mathbf{I}\right)^{-1} I_{o}^{T} d_{t}^{*}$, where $d_{t}^{*}$ is the distance of the training data. See for example [8] for a detailed description of ridge regression.

Table 1. Model parameters.

\begin{tabular}{|l|r|l|r|}
\hline Parameter & Value & Parameter & Value \\
\hline Radial lines $(n)$ & 8 & Sampling steps $(m)$ & 30 \\
Sampling distance (pixels) & 30 & Length constant $(c)$ & 0.35 \\
Gaussian blur - simulated $(\sigma)$ & 2 & Gaussian blur - real $(\sigma)$ & 1 \\
Radial similarty $(\eta$ - Eq. 2) & 1 & Variance weight $(\xi-$ Eq. 2$)$ & 4000 \\
Gaussian weight $(s$ - Eq. 3$)$ & $0.5 m$ & Variance weight $(\kappa$ - Eq. 6$)$ & 100 \\
\hline
\end{tabular}




\section{Experiments}

In this section we will experimentally show the performance of our particle model. We want to investigate the precision and accuracy of our model. By precision we mean how good our model is in predicting the true size, shape and particle depth. The accuracy refers to variation in the model predictions. The experiments are conducted in relation to size estimation, shape estimation and the particles distance to the focus plane. For these experiments we chose the parameter shown in Table 1. Furthermore, we investigate the robustness of the method in relation to the initial position and size estimates of the particles.

Data The endoscopic probe consists of three doublets with different powers separated as shown in Fig. 6. The distance between the object plane (particles) and the first optical element, which is a cover plate, is just $1 \mathrm{~mm}$. The separations between the optical elements up to the CCD is maintained and optimized to provide a magnification of 6 . The design is performed in Zemax optical design software. The total track length from object to image (particles to CCD) is $25 \mathrm{~cm}$ and the optical resolution of the system is 2 microns. The entire visible wavelength region is used to optimize this system $(480-650 \mathrm{~nm})$. The depth of focus at the object side is computed to be $+/-75$ microns when defined by a drop of more than $90 \%$ of the modulation transfer function. Modeling is done in a non-sequential mode in Zemax, which allows us to incorporate the real situation of illumination with back light of spherical and ellipsoidal particles. Using the non-sequential mode we are able to handle diffuse light and 3D particles. The diffuse light source is located a few millimeters behind the particles and emits light in the specified wavelength range randomly over a 15 degrees angle. The particles used are transparent with refractive index of 1.6 at $555 \mathrm{~nm}$ wavelength. Several million rays per simulation were used to generate a single image with particles. Imaging is done using a CCD array with 4 Megapixels of 7 micron pitch.

The real data set consists of particles in water suspension placed between two glass sheets, which have been moved with $\mu \mathrm{m}$ precision relative to the focus plane. $25 \mu \mathrm{m}$ particles are shown in Fig. 7 .

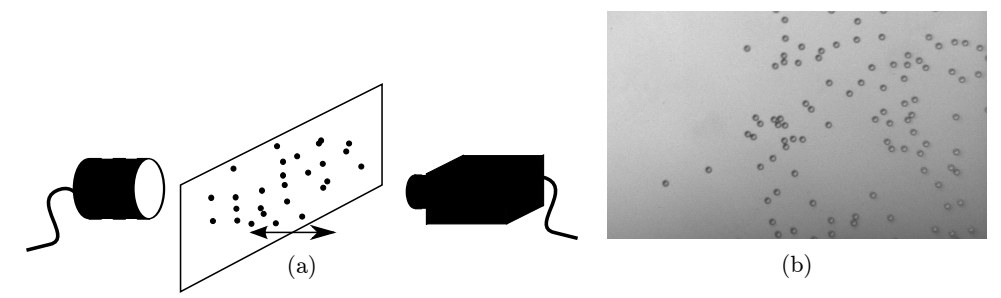

Fig. 7. Setup for acquiring real data. Particles are placed between glass sheets as illustrated in (a), which can be moved relative to the camera with $\mu$ m precision. In (b) an image example is shown with LED back illumination and $25 \mu \mathrm{m}$ spherical transparent particles. 

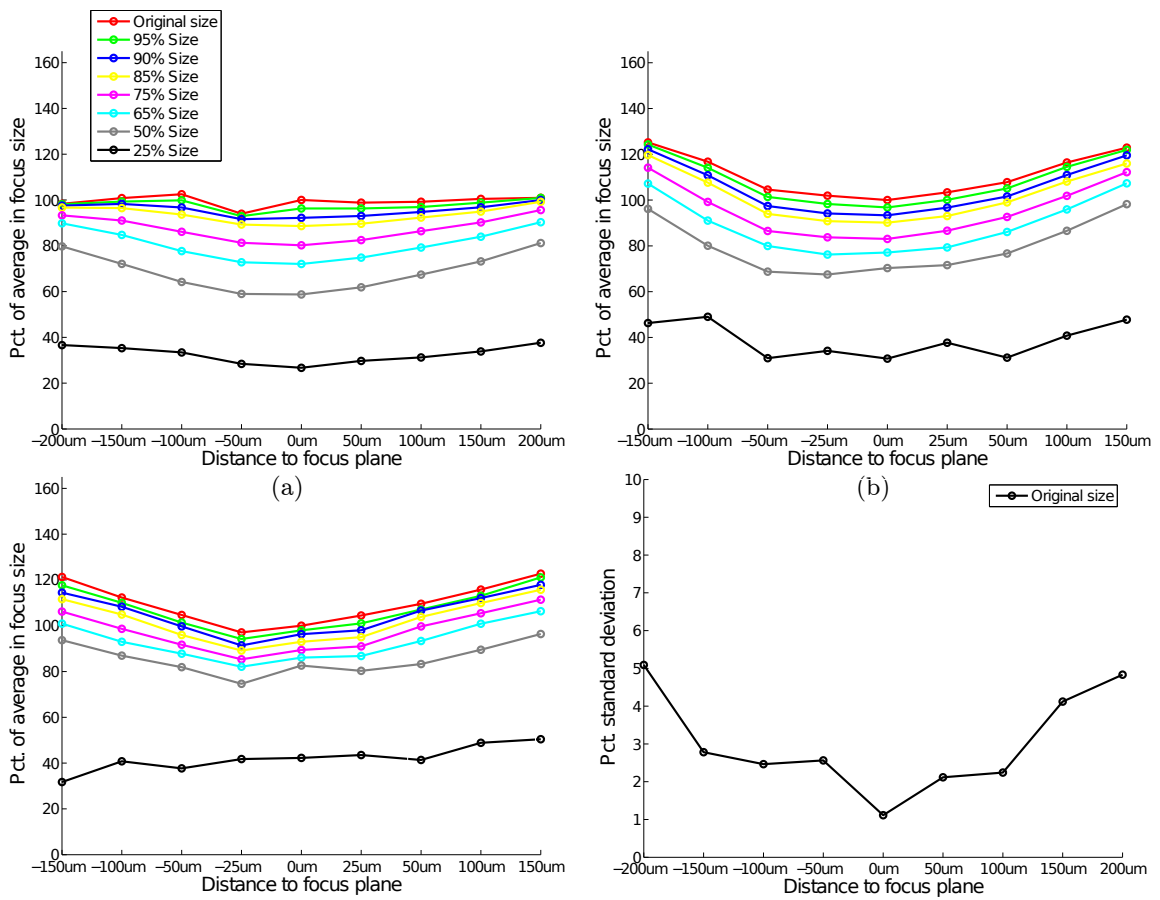

(c)

(d)

Fig. 8. Experiment with change of size. The horizontal axis of (a)-(c) shows the average radial distance relative to the in focus particle of original size. Standard deviation of the size estimate in percent of the original (d). Note the bias towards overestimation of size and less certainty as a function of out-of-focus.

Size experiment In this experiment we investigate the robustness of our size estimation. We have both tested the mean value and standard deviation of the estimated size, and how it depends on the distance to the focus plane. The results are shown in Fig. 8. The first three graphs (a)-(c) shows a relative size estimate as a function of distance to the focus plane, and each curve shows an individual size. There is a general bias towards overestimating the size of particles that are outof-focus and small particles are also somewhat overestimated. The model is not capable of handling very large size changes, and gives an erroneous prediction for particle scaled to $25 \%$ size. This is due to the fixed parameter setting where the sampling is too coarse to identify the small particles. Size variation is obtained by scaling the images.

Fig. 9 illustrates the robustness to inaccurate spatial initialization. The model will only fail in finding a good center approximation if it is initialized far from the particle and especially if it is done diagonally.

Shape experiment The purpose of this experiment is to investigate how the model deforms to adapt to non-spherical particles. We have conducted experiments on 

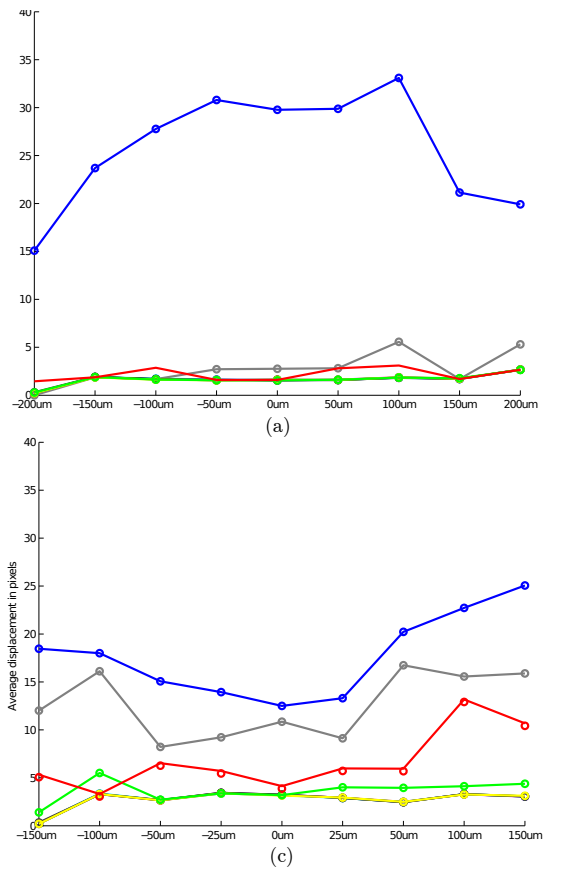
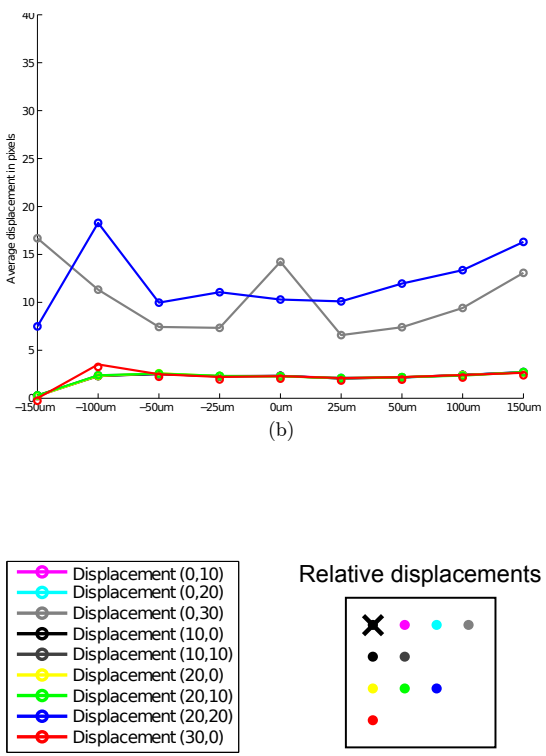

Relative displacements

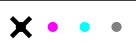

-

-

Fig. 9. Experiment for testing robustness to wrong spatial initialization of particle. The vertical axis is the average distance in pixels to the true position and the horizontal axis is the particle distance to the focus plane. A $50 \mu \mathrm{m}$ particle has a radius of about 17 pixels. Experiments have been carried out for simulated particles, which are spherical $(\mathrm{a})$, ellipsoids $(1 \times 2)(\mathrm{b})$, and ellipsoids $(1 \times 3)(\mathrm{c})$. Ellipsoids have the major axis vertical. The displacements are schematically shown in (b). Each displacement step is 10 pixels.

synthetic data with known ground truth and on real spray data. The spray data reflects some challenges encountered in particle analysis. The results of the synthetic data are shown in Fig. 10, where the relation between the horizontal and vertical line segments are plotted as a function of particle distance to the focus plane. The particles do not adapt completely to the expected shape, and there is a tendency for out-of-focus particles to be more circular than in focus particles. Despite the particle shape is not found exactly from the experiment, this can be inferred by regression, which we will show next. Examples of model alignment with real data is shown in Fig. 11.

Regression experiment Results from our regression experiment is shown in Table 2 . The regression is performed using ridge regression with $\lambda=10^{-5}$. We divided our data set into approximately half training and half test sets, which was 12 particles from the simulated set for training and 13 particles for test, from each image. In the real data set, we have 82 depicted particles, and the split was 41 in each group. We had 27 simulated images, giving 675 observations for the 


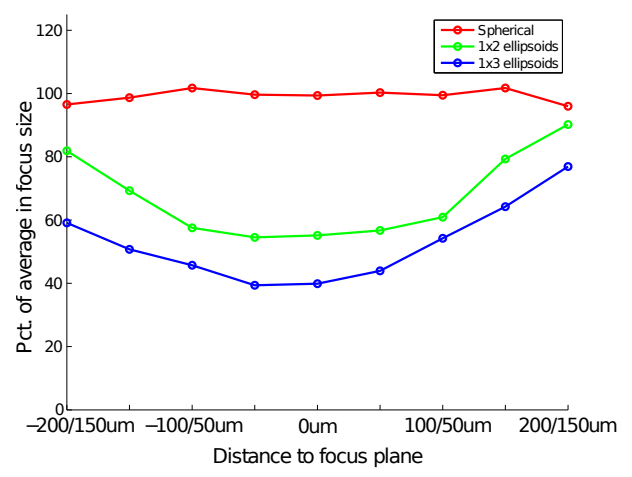

Fig. 10. Shape experiment. The horizontal axis is the relation between the vertical and the horizontal line segments from our particle model, corresponding to the minor and major axis in the simulated ellipsoids. The true relation for the red curve would be $100 \%$, the green curve would be $50 \%$ and the blue curve would be $33 \%$.

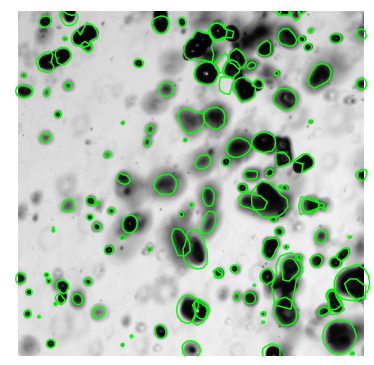

(a)

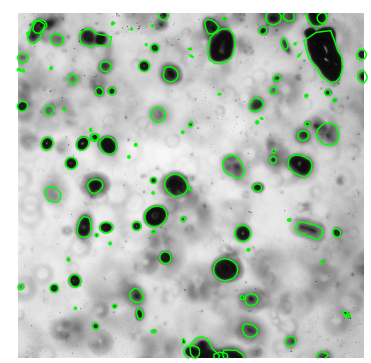

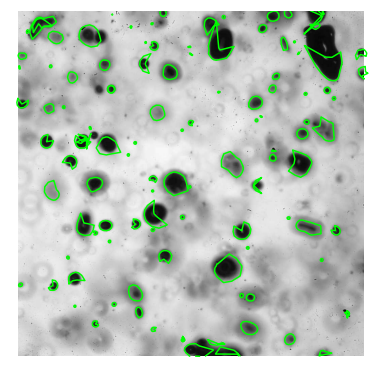

(b)

Fig. 11. Examples of model alignment with images of water sprays. The model aligns well to most water droplets including the ones that are out of focus. In (a) two examples from the new model formulation is shown, and results of the second example is shown in (b) based on the original model formulation [3]. It is clear that the new particle alignment procedure improves the result. The main problem is now particles initially detected as two blobs by the blob detector, so some of them has two or more models aligned to them.

simulation set. In the observed data set we have 82 particles in 9 images giving 738 observations. The results are obtained from 100 random splits in test and training data. We use the mean radial descriptor $\left(I_{o}\right)$ and the length for each line segment $\left(r_{o}\right)$ as input to our regression, see Eq. 7 .

In the simulated data we perform a regression for both distance to the focus plane, particle size, and shape, which is the ratio between the major and minor axis. The obtained results show precise predictions, indicating that this characterization is adequate for reliable particle modeling. For the real data we also obtained satisfactory prediction of the distance to the focus plane, but with about $50 \%$ lower precision, compared to the artificial data. 
Table 2. Regression model. Regression has been done for both simulated and real data. There were 25 particles in the simulated data and 82 particles in the real data set. The reported numbers are the standard deviation of the absolute errors of the regression, and the size range of the numbers. The columns are distance to the focus plane (Distance FP), average radial line length (Size), relation between the radial and horizontal line lengths (Shape).

\begin{tabular}{|c|c|}
\hline \multicolumn{2}{|r|}{ Simulated data } \\
\hline & \begin{tabular}{|ll} 
Distance FP & Size
\end{tabular} \\
\hline Std. & $14.20 \mu \mathrm{m} \quad 0.8921 \mu \mathrm{m} \quad 0.0357$ \\
\hline Range & $0-200 \mu \mathrm{m} 33.3-50 \mu \mathrm{m} 0.33-1.0$ \\
\hline \multicolumn{2}{|r|}{ Real data } \\
\hline & Distance FP \\
\hline Std. & $21.69 \mu \mathrm{m}$ \\
\hline Range & $0-180 \mu \mathrm{m}$ \\
\hline
\end{tabular}

\section{Discussion}

We have described a deformable model, based on radial intensity sampling, and shown how it can be used for inferring size, shape and distance to the focus plane for back illuminated particles. This information is useful for process inspection, where not only size and shape information is obtained, but also 3D distribution information.

The data for our experiment is based on LED illumination, both what is used in the real data, and what is simulated. This is a rather cheap solution, and if it can provide satisfactory results, it will be a cost effective solution. But the rather diffuse illumination from the LEDs could be replaced by collinear laser, which will give much higher particle contrast, and therefore potentially improved performance. Whether this will give larger depth of field or just improved predictions is for future investigations to show.

The size experiment illustrates how robust our particle model is to the initialization. With the same set of parameters, it is capable of handling up to $50 \%$ scale change. In the spray experiment we used scale space blob detection for initializing the particle model, and adaptiveness to scale change and spatial initialization worked fine for the precision of blob detection. The only problematic issue is when an elongated particle is detected as two or more blobs. If elongated particles are important in the analysis, then alternative detectors should be considered.

Scaling images for size variation does not account for the change in optical properties of smaller particles. We know that smaller particles in back-illumination change appearance caused by scattering effects like refraction and defraction, and this requires further investigations to verify that our model will be able to characterize these particles. The appearance change will result in blurred particles, which our particle model handles fine. The main focus should therefore be on whether the regression model can predict the true size. Our regression experiment indicates that this should be possible. 
The shape experiment shows that the model does not adapt precisely to the shape of the particle. This is caused by the Gaussian noise removal, which also blurs the particles making them appear less ellipsoid than they are in reality. The reason for using Gaussian convolution, which actually acts contrary to the deconvolution that we are trying to infer, is the noise level in the simulations. The noise is much larger, than what is seen in the real data, which can be seen by comparing the images in Fig. 6 (c) and Fig. 7 (b). But even with this high noise level, it was possible to infer the true shape by ridge regression.

Our regression experiment shows that the size, shape, and distance to the focus plane can be inferred using our particle model. This is highly encouraging, because it can help in performing more reliable particle analysis, than by just using the in focus particles, see e.g. [7]. The linear ridge regression is a simple procedure, and much more advanced methods exists, which for example can handle non-linearities. This can be relevant for inferring particle information of a larger size range or very small particles, where scattering effects are more pronounced. In this paper we have chosen to primarily focus on the particle model, so we leave this for future investigations.

There are no comparative studies between our model and similar approaches, because other procedures are based on modeling in focus particles, see e.g. [6, 7]. The radial sampling lines, which we use in our model, will give much weight to the center part of the particle.

\section{Conclusion}

This paper builds on previous work where the reliable method for obtaining information about particle size, shape and distance to the focus plane was shown. The main contributions of this paper is an extended and more robust particle model, more details on the model and demonstration on challenging real data of spray droplets. Through this work we have shown that important particle information can be obtained from 2D images with narrow depth of field. This includes size, shape and volumetric information about particle distribution. The principle of the characterization is simple radial samples, which encodes all the necessary information.

\section{Acknowledgements}

This work has been partly financed by the EU-project PROVAEN under the Sixth Framework Programme. We also thank our collaborators from Dantec $\mathrm{A} / \mathrm{S}^{4}$ for providing data and fruitful discussions.

\footnotetext{
${ }^{4}$ http://www.dantecdynamics.com/
} 


\section{References}

1. Blaisot, J., Yon, J.: Droplet size and morphology characterization for dense sprays by image processing: application to the Diesel spray. Experiments in Fluids 39(6), 977-994 (2005)

2. Cho, S., Matsushita, Y., Lee, S., Postech, P.: Removing non-uniform motion blur from images. In: IEEE 11th International Conference on Computer Vision, 2007. ICCV 2007. pp. 1-8 (2007)

3. Dahl, A., Jørgensen, T., Gundu, P., Larsen, R.: Shape and Size from the Mist - a Deformable Model for Particle Characterization. In: International Conference on Computer Vision Theory and Applications 2010 (2010)

4. Dai, S., Wu, Y.: Motion from blur. In: Proc. Conf. Computer Vision and Pattern Recognition. pp. 1-8 (2008)

5. Fantini, E., Tognotti, L., Tonazzini, A.: Drop size distribution in sprays by image processing. Computers \& chemical engineering 14(11), 1201-1211 (1990)

6. Fisker, R., Carstensen, J.M., Hansen, M.F., Bødker, F., Mørup, S.: Estimation of nanoparticle size distributions by image analysis. Journal of Nanoparticle Research 2(3), 267-277 (2000)

7. Ghaemi, S., Rahimi, P., Nobes, D.: Measurement of Droplet Centricity and Velocity in the Spray Field of an Effervescent Atomizer. Int Symp on Applications of Laser Techniques to Fluid Mechanics, Lisbon, Portugal, 07-10 July, 2008 (2008)

8. Hastie, T., Tibshirani, R., Friedman, J., Franklin, J.: The elements of statistical learning: data mining, inference and prediction. The Mathematical Intelligencer $27(2), 83-85(2005)$

9. Kundur, D., Hatzinakos, D.: Blind image deconvolution. IEEE signal processing magazine 13(3), 43-64 (1996)

10. Lecuona, A., Sosa, P., Rodriguez, P., Zequeira, R.: Volumetric characterization of dispersed two-phase flows by digital image analysis. Measurement Science and Technology 11, 1152 (2000)

11. Lee, S., Kim, Y.: Sizing of spray particles using image processing technique. Journal of Mechanical Science and Technology 18(6), 879-894 (2004)

12. Levin, A.: Blind motion deblurring using image statistics. Advances in Neural Information Processing Systems 19, 841 (2007)

13. Lindeberg, T.: Scale-space theory in computer vision. Springer (1994)

14. Lucy, L.B.: An iterative technique for the rectification of observed distributions. The astronomical journal 79(6), 745-754 (1974)

15. Narayan, R., Nityananda, R.: Maximum entropy image restoration in astronomy. Annual review of astronomy and astrophysics 24(1), 127-170 (1986)

16. Richardson, W.H.: Bayesian-based iterative method of image restoration. Journal of the Optical Society of America 62(1), 55-59 (1972)

17. Shan, Q., Jia, J., Agarwala, A.: High-quality motion deblurring from a single image. ACM Transactions on Graphics-TOG 27(3), 73-73 (2008)

18. Shan, Q., Xiong, W., Jia, J.: Rotational motion deblurring of a rigid object from a single image. In: IEEE 11th International Conference on Computer Vision, 2007. ICCV 2007. pp. 1-8. Citeseer (2007)

19. Starck, J.L., Pantin, E., Murtagh, F.: Deconvolution in astronomy: a review. Publications of the Astronomical Society of the Pacific 114(800), 1051-1069 (2002)

20. Wiener, N.: Extrapolation, Interpolation, and Smoothing of Stationary Time Series. The MIT Press (1964) 